\title{
Age Advantages in Emotional Experience Persist Even Under Threat From the COVID-19 Pandemic:
}

\section{Supplemental Material}

\section{Supplemental Results}

\section{R Packages}

Analysis was conducted using R version 3.6.1 (R Core Team, 2019) with packages pander (Daróczi \& Tsegelskyi, 2018), knitr (Xie, 2019), memisc: Martin Elff (2020), sjPlot (Lüdecke, 2020), sjmisc (Lüdecke, 2018), sjlabelled (Lüdecke, 2020), expss (Demin, 2020), tidyverse (Wickham, 2017), car (Fox \& Weisberg, 2019), psych (Revelle, 2018), GGally (Schloerke et al., 2018), Ime4 (Bates et al., 2015) ,and ImerTest (Kuznetsova et al., 2017).

Intensity of Emotions

Participants reported experiencing emotions with different levels of intensity, when these emotions were felt. Repeated measures ANOVAs revealed significant differences for emotions intensity within positive emotions $\left(F_{\text {within }}(15,13392)=95.85, p<.001\right)$ and negative emotions $\left(F_{\text {within }}(12,8332)=\right.$ $168.2, p<.001)$ alike. See Table S1 for mean intensities of each emotion.

\section{Age Correlations with Emotions}

As a whole, simple correlations of age with the frequencies of positive and negative emotions were not significantly different in strength (Fisher's $Z=1.83, p=.07)^{1}$. However, age was more strongly associated with reported intensity of negative, compared to positive, emotions (Fisher's $Z=3.35, p<$ .001). Within each valence, there was variability in the strength of association between age and the

\footnotetext{
${ }^{1}$ When accounting for personality, age was more strongly associated with the frequency of negative emotions $(r(936)=-.19, p<.001)$ than with that of positive emotions $(r(936)=0.07, p=.03$; Fisher's $Z=2.65, p=.008)$.
} 
reported frequency and intensity of discrete emotions (see Figure S1). Age was negatively associated most strongly with the frequencies of feeling bored, ashamed, and guilty. Age was most strongly positively associated with the frequency of feeling interested.

\section{Mixed-effects Models Results}

Variability in the strength of age associations with the frequencies and intensities of discrete emotions might suggest that the observed age relationships reported in the main text are driven by specific emotions. To ensure that the observed relationship is not driven solely by specific emotions and to assess the generalizability of our findings, we also ran the final regression model, with all covariates, as a mixed-effects model with random age slopes for specific emotions. As can be seen in Table S2, the effect of age remained significant, suggesting that even though age is more strongly associated with reporting a lower frequency of certain negative emotions, the overall pattern is generalizable across them. The same is true for the association between age and higher frequency of positive emotions, suggesting that both patterns are generalizable across individual positive and negative emotions in terms of emotional frequency.

When we used the same mixed-effects models approach for reported intensities of emotions, the association between age and reporting lower intensity of negative emotions remained significant. Though age is not a significant predictor of the intensity of positive emotions in the mixed-effects model, this is due to the inclusion of personality covariates (as described in the main text). When random age slope for specific emotions was entered to the model predicting the reported intensity of positive emotions by age alone, the relationship was significant $(B=0.05, \mathrm{t}(16.06)=2.56, p=.02)$.

\section{Age and Gender Interactions}


As reported in the main text, the majority of our age effects held across gender, with a significant age-gender interaction only for frequency of positive emotions. We found that age is associated with reporting feeling negative emotions less frequently regardless of gender (Age x Gender interaction: $\beta=.05, p=.18)$, and gender interactions did not alter the association between age and reported intensity of negative emotions. (Age $x$ Gender interaction: $\beta=.04, p=.37$ ). Similarly, the interaction between age and gender was not significant in the model predicting self-reported intensity of positive emotions $(\beta=-.04, p=.25)$. 


\section{Supplemental References}

Bates, D., Maechler, M., Bolker, B., \& Walker, S. (2015). Lme4: Linear mixed-effect models using eigen and S4. R package version 1.1-23. https://cran.r-project.org/package=Ime4

Daróczi, G., \& Tsegelskyi, R. (2018). Pander: An R pandoc writer. R package version 0.6.3. https://cran.rproject.org/package=pander

Demin, G. (2020). Expss: Tables, labels and some useful functions from spreadsheets and SPSS Statistics. R package version 0.10.2. https://cran.r-project.org/package=expss

Elff, M. (2020). Memisc: Management of survey data and presentation of analysis results. $\mathrm{R}$ package version 0.99.22. https://cran.r-project.org/package=memisc

Fox, J., \& Weisberg, S. (2019). An R companion to applied regression. Sage publications.

Kuznetsova, A., Brockhoff, P., \& Christensen, R. (2017). ImerTest package: Tests in linear mixed effects models. Journal of Statistical Software, 82(13), 1-26. http://doi.org/10.18637/jss.v082.i13

Lüdecke, D. (2018). Sjmisc: Data and variable transformation functions. R package version 2.8.5. $\underline{\text { https://cran.r-project.org/package=simisc }}$

Lüdecke, D. (2020). Sjlabelled: Labelled data utility functions. R package version 1.1.4. https://cran.rproject.org/package=sjlabelled

Lüdecke, D. (2020). SjPlot: Data visualization for statistics in social science. R package version 2.8.3. https://cran.r-project.org/package=sjPlot

R Core Team. (2019). R: A language and environment for statistical computing. R Foundation for Statistical Computing. https://www.R-project.org

Revelle, W. (2018) Psych: Procedures for personality and psychological research. R package version 1.8.12. https://cran.r-project.org/package=psych. 
Schloerke, B., Crowley, J., Cook, D., Briatte, F., Marbach, M., Thoen, E., Elberg, A., \& Larmarange, J. (2018). GGally: Extension to ggplot2. R package version 1.4.0. https://cran.rproject.org/package=GGally

Wickham, H. (2017). Tidyverse: Easily install and load the tidyverse. R package version 1.2.1. https://cran.r-project.org/package=tidyverse

Xie, Y. (2019). Knitr: A general-purpose package for dynamic report generation in R. R package version 1.25. https://cran.r-project.org/package=knitr 


\section{Supplemental Tables}

Table S1

Mean Intensities of Emotions

\begin{tabular}{|c|c|c|c|}
\hline & $M$ & $S D$ & $95 \% \mathrm{Cl}$ \\
\hline \multicolumn{4}{|c|}{ Positive Emotions ( $N=945)$} \\
\hline Appreciative $^{a}$ & 2.36 & 1.01 & {$[2.30,2.42]$} \\
\hline Quiet $^{\mathrm{ab}}$ & 2.28 & 0.95 & {$[2.22,2.34]$} \\
\hline Calm ${ }^{b}$ & 2.21 & 0.93 & {$[2.15,2.27]$} \\
\hline Interested $^{\mathrm{b}}$ & 2.17 & 0.87 & {$[2.11,2.22]$} \\
\hline Content $^{c}$ & 2.03 & 0.93 & {$[1.97,2.09]$} \\
\hline Relaxed $^{c}$ & 2.03 & 0.92 & {$[1.97,2.08]$} \\
\hline Happy ${ }^{c}$ & 2.00 & 0.86 & {$[1.95,2.06]$} \\
\hline Peaceful cd & 1.97 & 0.92 & {$[1.91,2.03]$} \\
\hline Amused ${ }^{c d}$ & 1.97 & 0.91 & {$[1.91,2.03]$} \\
\hline Affectionate de & 1.87 & 0.95 & {$[1.81,1.93]$} \\
\hline Accomplished ef & 1.80 & 0.89 & {$[1.75,1.86]$} \\
\hline Proud ${ }^{\text {efg }}$ & 1.75 & 0.99 & {$[1.69,1.81]$} \\
\hline Energetic $^{\mathrm{fg}}$ & 1.73 & 0.86 & {$[1.68,1.79]$} \\
\hline Relieved ${ }^{\text {gh }}$ & 1.70 & 0.88 & {$[1.64,1.75]$} \\
\hline Joyful gh & 1.69 & 0.92 & {$[1.63,1.75]$} \\
\hline Excited $^{\mathrm{h}}$ & 1.61 & 0.85 & {$[1.56,1.66]$} \\
\hline \multicolumn{4}{|c|}{ Negative Emotions ( $N=942$ ) } \\
\hline Concerned $^{\mathrm{a}}$ & 2.23 & 0.98 & {$[2.16,2.29]$} \\
\hline Frustrated ${ }^{b}$ & 2.12 & 1.07 & {$[2.05,2.19]$} \\
\hline Anxious/worried ${ }^{b c}$ & 2.10 & 1.06 & {$[2.03,2.16]$} \\
\hline Bored $^{\mathrm{bc}}$ & 2.05 & 1.13 & {$[1.98,2.13]$} \\
\hline Irritated ${ }^{c d}$ & 2.00 & 1.06 & {$[1.93,2.07]$} \\
\hline Lonely de & 1.95 & 1.16 & {$[1.88,2.03]$} \\
\hline
\end{tabular}




$\begin{array}{lccc}\text { Sad }^{\mathrm{e}} & 1.87 & 1.06 & {[1.80,1.93]} \\ \text { Angry }^{\mathrm{f}} & 1.66 & 0.98 & {[1.60,1.72]} \\ \text { Disgusted }^{\mathrm{f}} & 1.61 & 1.11 & {[1.54,1.68]} \\ \text { Fearful }^{\mathrm{f}} & 1.59 & 0.99 & {[1.53,1.65]} \\ \text { Guilty }^{\mathrm{g}} & 1.32 & 0.92 & {[1.26,1.38]} \\ \text { Ashamed }^{\mathrm{g}} & 1.17 & 0.92 & {[1.11,1.23]} \\ \text { Embarrassed }^{\mathrm{g}} & 1.12 & 0.88 & {[1.06,1.17]}\end{array}$

Note: Within each valence, emotions that share a superscript do not differ significantly in a paired ttest at the alpha $=0.05$ level, after a Bonferroni correction for multiple comparisons. 


\section{Table S2}

Mixed-effects Linear Models Predicting Emotional Well-being by Age and Covariates

\begin{tabular}{|c|c|c|c|c|c|c|c|c|c|c|c|c|}
\hline \multirow{4}{*}{$\begin{array}{l}\text { Fixed Effects: } \\
\text { (Intercept) }\end{array}$} & \multirow{2}{*}{\multicolumn{3}{|c|}{$\begin{array}{l}\text { Frequency: } \\
\text { Negative Emotions }\end{array}$}} & \multirow{2}{*}{\multicolumn{3}{|c|}{$\begin{array}{l}\text { Frequency: } \\
\text { Positive Emotions }\end{array}$}} & \multicolumn{3}{|c|}{ Intensity: } & \multicolumn{3}{|c|}{ Intensity: } \\
\hline & & & & & & & \multicolumn{3}{|c|}{ Negative Emotions } & \multicolumn{3}{|c|}{ Positive Emotions } \\
\hline & \multirow{2}{*}{$\begin{array}{c}B \\
1.42^{* * *}\end{array}$} & \multicolumn{2}{|c|}{$95 \% \mathrm{Cl}$} & \multirow{2}{*}{$\begin{array}{l}B \\
2.03^{* * *}\end{array}$} & \multicolumn{2}{|c|}{$95 \% \mathrm{Cl}$} & \multirow{2}{*}{$\begin{array}{l}B \\
1.63^{* * *}\end{array}$} & \multicolumn{2}{|c|}{$95 \% \mathrm{Cl}$} & \multirow{2}{*}{$\begin{array}{l}B \\
1.92^{* * *}\end{array}$} & \multicolumn{2}{|c|}{$95 \% \mathrm{Cl}$} \\
\hline & & 1.08 & 1.75 & & 1.86 & 2.20 & & 1.38 & 1.88 & & 1.80 & 2.04 \\
\hline Age & $-0.12^{* *}$ & -0.19 & -0.04 & $0.06^{*}$ & 0.01 & 0.11 & $-0.18^{* * *}$ & -0.26 & -0.11 & -0.03 & -0.07 & 0.01 \\
\hline Risk to self & $0.09^{* * *}$ & 0.08 & 0.11 & $-0.05^{* * *}$ & -0.07 & -0.03 & $0.11^{* * *}$ & 0.08 & 0.13 & $-0.04^{* * *}$ & -0.05 & -0.02 \\
\hline Risk of complications & $0.05^{* * *}$ & 0.03 & 0.08 & $0.03^{* *}$ & 0.01 & 0.05 & $0.07^{* * *}$ & 0.04 & 0.09 & $0.06^{* * *}$ & 0.04 & 0.08 \\
\hline Openness & 0.002 & -0.00 & 0.03 & 0.01 & -0.02 & 0.01 & $0.07^{* * *}$ & 0.05 & 0.09 & 0.01 & -0.01 & 0.03 \\
\hline Conscientiousness & $-0.05^{* * *}$ & -0.07 & -0.03 & $0.03^{* * *}$ & 0.02 & 0.05 & $-0.05^{* * *}$ & -0.07 & -0.03 & $0.03^{* *}$ & 0.01 & 0.04 \\
\hline Extraversion & $0.02^{*}$ & 0.00 & 0.04 & $0.04^{* * *}$ & 0.03 & 0.05 & 0.002 & -0.02 & 0.02 & $0.03^{* *}$ & 0.01 & 0.04 \\
\hline Agreeableness & $-0.02^{*}$ & -0.04 & 0.00 & $0.05^{* * *}$ & 0.03 & 0.06 & 0.02 & -0.01 & 0.04 & $0.06^{* * *}$ & 0.05 & 0.08 \\
\hline Emotional Stability & $-0.30^{* * *}$ & -0.32 & -0.28 & $0.20^{* * *}$ & 0.19 & 0.22 & $-0.29^{* * *}$ & -0.31 & -0.27 & $0.18^{* * *}$ & 0.16 & 0.20 \\
\hline Race (White) ${ }^{a}$ & -0.02 & -0.06 & 0.02 & $-0.05^{* *}$ & -0.08 & -0.02 & $0.05^{*}$ & 0.00 & 0.1 & 0.02 & -0.02 & 0.05 \\
\hline Health & -0.005 & -0.02 & 0.02 & $0.10^{* * *}$ & 0.09 & 0.12 & 0.007 & -0.03 & 0.02 & $0.12^{* * *}$ & 0.10 & 0.14 \\
\hline Education Level & 0.005 & -0.01 & 0.02 & $-0.03^{* * *}$ & -0.05 & -0.02 & 0.003 & -0.02 & 0.02 & $-0.02^{* *}$ & -0.04 & -0.01 \\
\hline Employed (Yes) ${ }^{a}$ & 0.01 & -0.02 & 0.05 & 0.01 & -0.02 & 0.04 & -0.03 & -0.07 & 0.01 & 0.01 & -0.02 & 0.04 \\
\hline Live alone (Yes) ${ }^{a}$ & -0.02 & -0.05 & 0.02 & $-0.07^{* * *}$ & -0.10 & -0.03 & $0.15^{* * *}$ & 0.10 & 0.20 & 0.01 & -0.03 & 0.05 \\
\hline Random Effects: & (variance) & & & (variance) & & & (variance) & & & (variance) & & \\
\hline
\end{tabular}




\begin{tabular}{lllll}
\hline Emotions: Intercepts & 0.305 & 0.095 & 0.162 & 0.049 \\
Emotions: Age slopes & 0.014 & 0.007 & 0.013 & 0.004 \\
Residual variance & 0.762 & 0.651 & 0.913 & 0.761 \\
\hline
\end{tabular}

Note. Continuous predictors were standardized. Statistical significance was obtained through t-tests using the Satterthwaite method.

${ }^{a}$ Parentheses denote values of binary variables coded as 1.

${ }^{*} p<.05 .{ }^{* *} p<.01 .{ }^{* * *} p<.001$. 


\section{Supplemental Figures}
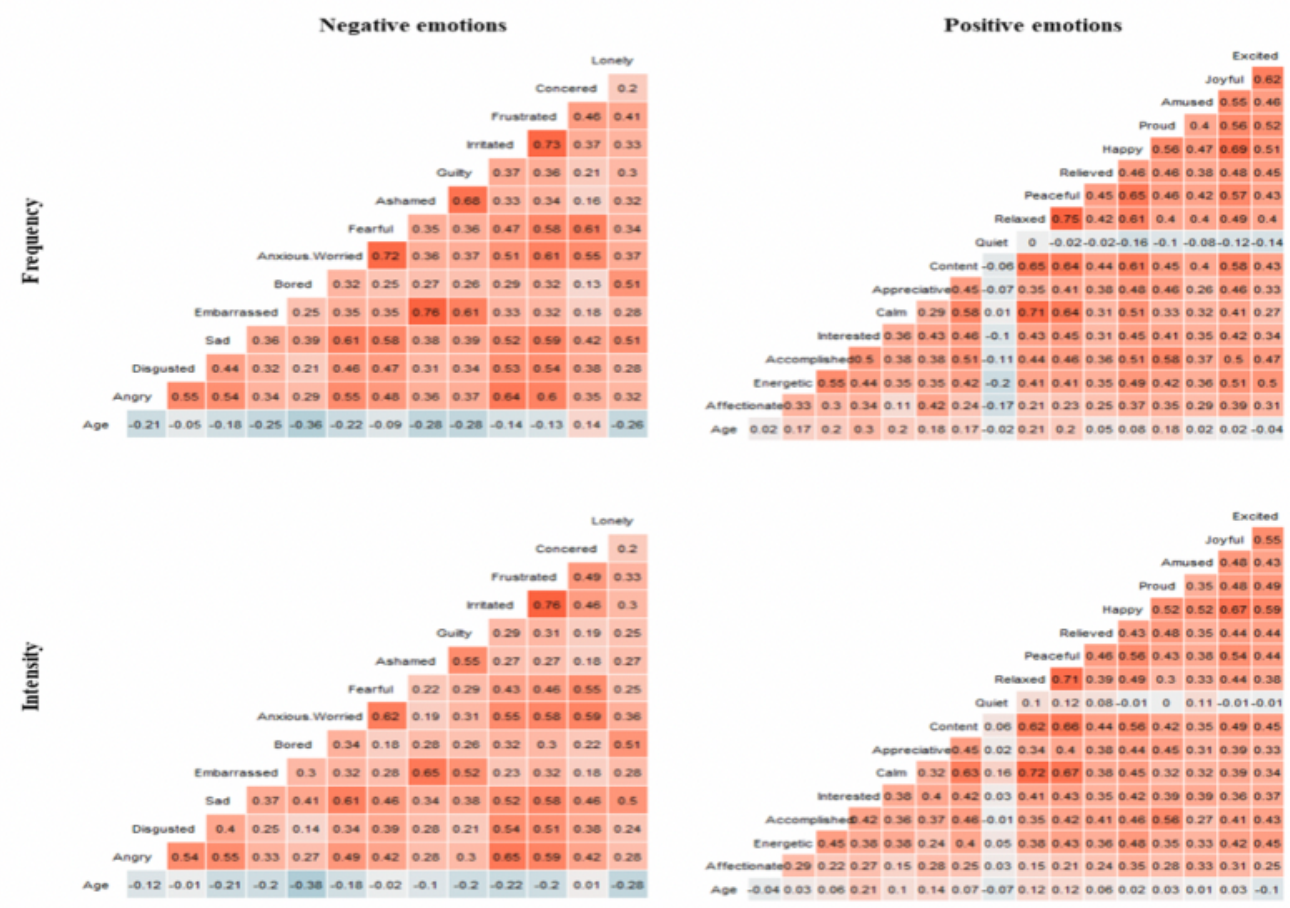

Fig. S1. Correlations of emotions with age, by valence and measure. Correlations with $|<=0.06|$ are not significant with alpha $=.05$. Correlations with $|<=0.08|$ are not significant with alpha $=.01$. 